\title{
اختلافات دراسة علم الأصهوات بين العربية واللسانيات الحديثة
}

\section{Eva Lathifah Fauzia}

UIN Sunan Gunung Djati

(bundafaiq.elf@gmail.com)



\begin{abstract}
ABSTRAK
Kata Kunci:

Branches of phonology

Modern Linguistics

Arabic Voices

The aim of this study is to describe the diversity of phonology and the field differences between the study of Arabic phonology and general phonology. After examining the diversity of these branches on the science of sounds, the researcher will show the divisions and features of Arabic phonology among others. Finally, this research examines the utilization of these sciences and how to apply the teaching of sounds to other speakers.
\end{abstract}

Eva Lathifah Fauzia

اختلافات دراسة علم الأصوات بين العربية واللسانيات الحديثة
ALSUNA Vol. 2 (1), 2019

http://e-journal.ikhac.ac.id/index.php/alsuna https://doi.org/10.31538/alsuna 
مما لاشك أن الإنسان قد عرف الكلام المنطوق أولا قبل أن يخترع الكتابة. مهما كانت الكتابة تمثيلا للنطق فإنها لا تستطيع نقل حركات الجسم وتعبيرات الوجه ونغمات الأصوات وسائر الملامح السيميائية للكلام. فالكلام هو نطق صوت الجمل التي تتكون من الكلمات والصوت عملية حركية يقوم بها الجهاز النطقي وتصحبها آثار سمعية معينة تأتي من تحريك الهوى فيما بين مصدر إرسال الصوت وهو الجهاز النطقي ومزكز استقباله وهو الأذن(تمام حسان، 77). من المعروف إن الصهوت عنصر من عناصر اللغة فلا يسمي باللغة اذا ليست فهها أصواتا لها المعنى.فلذا يعرف ابن الجني تعريفا على أن اللغة هي أصهوات يعبر بها كل قوم عن أغراضهم (وافي، Y10). فترجع أهم ظواهر اللغة إلى قسمين رئيسيين : الظواهر المتعلقة بالصوت والظواهر المتعلقة بالدلالة وهذان الناحيتين في تطور مطرد وتغير مستمروهي في تطورها تتأثر بعوامل شتى وتخضع لطائفة كبيرة من القوانين(وافي، Y10). انطلاقا من ذلك، في هذه المقالة نحدد البحث إلى الإطار النظري الذي يتكون من تطورات علم الأصوات و تعريف علم الأصيوات وتفريعه، و نظريات علم الأصيوات والآخر البحث عن الاستفادة من علم الأصهوات في تعليم اللغة العربية. فروع علم الأصوات وقد يتفرع علم الأصهوات إلى فروع كثيرة. و حاول اللغويون تصنيف هذا العلم على أساس تناوله من جوانب الصوت. والتصنيفات الشائعة لعلم الأصوات كما يلى :

\section{1. تصنيف علم الأصوات من ناحية ماديتها ووظيفتها}



وقد عرّف نصر الدين إدريس جوهر أنه علم يدرس الأصهوات فيزائيا وعضويا من






الجهاز الصوتي ووقفا لصفاتها التي تميز كل صوت منها عن غيره عند صدوره من هذا


وظائفها اللغوية أو تحديد اللغة التي تنتهي إلهيا (قدّور، . ع).

Eva Lathifah Fauzia

اختلافات دراسة علم الأصوات بين العربية واللسانيات الحديثة 
و يبحث علماء اللغة عن دراسات الفونيتيك عن الجوانب الآتية :أعضاء النطق، وانتاج الصوت اللغوي ( كيف ينطق )، وتصنيف الصوامت و تصنيف الصوائت (الراشدي،ه). و أما عند عادل خالف هي وصف الجهاز الصوتي، عدد أصوات اللغة

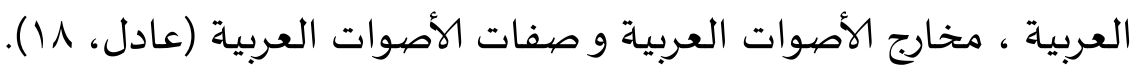
r. ( ب الفونيماتيك

هو علم الأصوات الموظفة ههتم بدراسة الصيوت اللغوي داخل البنية، أي من حيث علاقته بالأصوات الأخرى من ناحية، والمعنى أو وظيفة الصوت في تحديد المواته المعنى من ناحية أخرى (الراشدي،7). و مناسبا بقول نصر الدين أنه علم يدرس الأصهوات وظيفيا داخل التركيب لغة معينة من حيث خصائصها، وصفاتها ووظيفتها الدلالية. ويعرّف باعتبار أخر الخر التران أنه علم هيتم بالصوتيات الوظيفية واقتصرا لذلك على دراسات الوحدات الصهوتية

$$
\text { فيسهى بعلم الأصيوات الوظيفي (غانم، . ع). }
$$

إن الفونيم المعادل النفسي للصوت. ولا تستعمل كل اللغة نفس الوحدات الصهوتية التي تستعملها لغة أخرى لكي تركب منها الكلمات، وإنما تستعمل كل لغة وحدات صوتية مختلفة، وهذه الوحدات الصوتية تسمى الفونيمات و دراسة هذه هي الفونيماتيك و كيف تركيبها واتصالها بعضها بعضا وعلاقتها بالمقاطع والنبر وغير ذلك (كمل بشر، . 1). ولذلك أن فونيتك يبحث عن كيفية نطق الأصوات بغير نظر إلى وظيفته

$$
\text { فونيماتيك يدرس فيه الأصوات بنظر إلى وظيفته. }
$$

r. تصنيف علم الأصوات من ناحية المنهج المتبع لدراسة الأصوات، وتندرج تحت هذا

$$
\begin{aligned}
& \text { التصنيف ثلاث تفريعات لعلم الأصوات هي (إدريس جوهر، با بـr ): } \\
& \text { 1.) تقسيم إلى علم الأصهوات الوصفي وعلم الأصهوات المعياري }
\end{aligned}
$$

أ- علم الأصوات الوصفي وهو يصف أصوات اللغة المعينة كما ينطقها أهلهافي بيئتها

$$
\text { الخاصية وذلك بطريقة موضوعية دون اللجوء إلى التحليل والتأويل. }
$$



تحديد قواعد وضوابط لها يمكن الاعتماد عليها والتحكم بها في نطق هذه اللغة.

فعلم الأصوات الوصفي يصف الأصوات كما تنطق أهلها وأما علم الأصوات

$$
\text { r.) تقسيم إلى علم الأصيوات السنكرونيك وعلم الأصوات الميوات الديكرونيك أي كما يجب أن تنطق. }
$$

Eva Lathifah Fauzia

اختلافات دراسة علم الأصوات بين العربية واللسانيات الحديثة
ALSUNA Vol. 2 (1), 2019 
أ- علم الأصوات السنكرونيك وهو يدرس أصوات اللغة المعينة خلال مدة زمنية

$$
\text { محددة ويسمي أيضا بعلم الأصوات التزامني أو علم الأصهوات المتزامن. }
$$

ب- علم الأصوات الديكرونيك، وهو يدرس أصوات اللغة المعينة من حيث تطورها وما

$$
\text { طرأ علية من تغيرات عبر العصور والأزمنة. }
$$

$$
\text { r.) تقسيم إلى علم الأصوات المقارن وعلم الأصوات التقابلي }
$$



\section{الأسرة لغوية واحدة. مثال مقارنة بين اللغة الانجليزية و الفرنسية.}





$$
\text { العربية والإندونيسية. }
$$

\section{r. تصنيف علم الأصوات من ناحية ميسرة الأصوات في عملية الكلام



وهو يدرس الأصوات من جانها النطقي و الفسيولوجى المتماثلان في عملية النطق



$$
\text { r. الاصوات. علم الأصهوات الأكوستكيو }
$$

وهو يدرس الأصوات من جانها الفيزيائي أو الأكوستيكي المتمثل في الموجات




السامع. فموضوع الدراسة هذا العلم : سعة الصهوت ودرجة الصهوت ونوع الصيوت.



وهو يدرس ميكانيكيةالجهاز السمعي وتأثره بالأصوات من حيث تموجاتها، و



ويقسم أحمد محمد قدور الفونيتيك أي علم الأصهوات إلى أربعة أقسام مناسبا



1) علم الأصوات النطقي أو الفيزيولوجي : يدرس فيه محتى مخارج الأصوات الكلامية وطريقة

$$
\text { نطقها، ويبين أعضاء النطق ويصف عملها ويصنف صهاتها. }
$$

Eva Lathifah Fauzia

اختلافات دراسة علم الأصوات بين العربية واللسانيات الحديثة

ALSUNA Vol. 2 (1), 2019 
r) علم الأصوات الفيزيائي أو السمعي : يدرس فيه الموجات الصوتية الصادرة عن جهاز





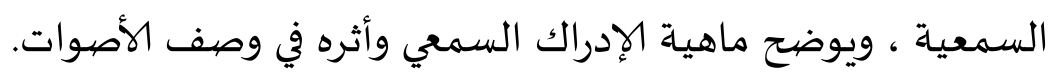

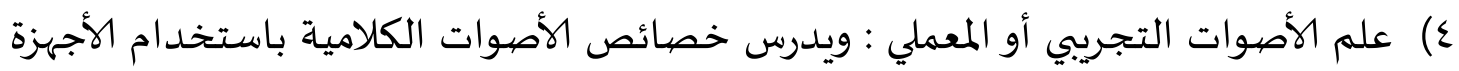



\section{نظريات علم الأصهوات العربية}

وفي هذا الصدد نبحث عن هذه النظريات اعتمادا على مواد علم الأصوات النطقي وعلم الأصوات التشكيلى . فالمواد لعلم الأصوات النطقي هي وصف جهاز النطق و مخارجها وصفاتها و الأصوات الصائتة والصامتة. أما علم الأصوات التشكيلي أو الفونولوجيا التركيبي هو الذي ينتقل من الجزء إلى الكل فالبحث فياء عن الفونيم والمقطع و مجموعة النبر و مجموعة النغمية و المجموعة النفسية والجملة الفونولوجيا

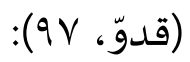

$$
\text { 1. 1. وصف جهاز النطق }
$$

تحدد الدراسات الحديثة جهاز النطق بدءا من الرئتين وانتهاء بالشفتين، وتميز بين

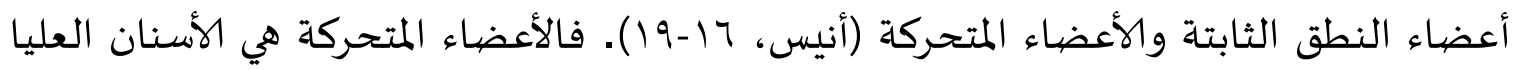





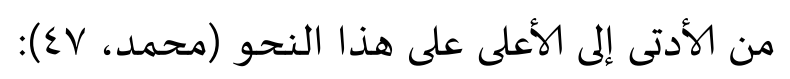





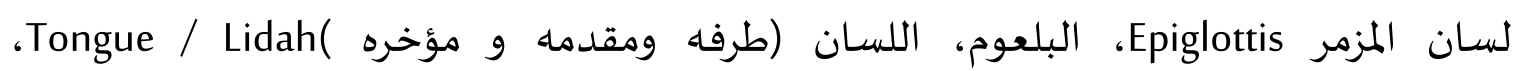



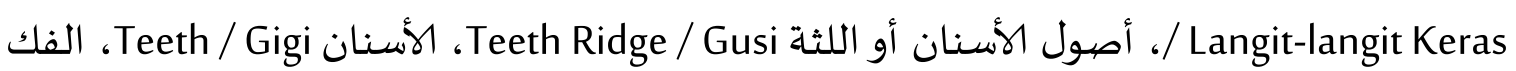



$$
\text { r. ) وصف الأصوات العربية الأصهوات من ناحية مخارجها }
$$

Eva Lathifah Fauzia

اختلافات دراسة علم الأصوات بين العربية واللسانيات الحديثة 
المخرج هو مكان النطق الذي يحدث فيه التصويت. إن عدد مخارج الأصوات

العربية عند المتقدمين هي ستة عشر كما قاله إبن الجني. وأما المحدثين يرون أهها عشرة كما قال

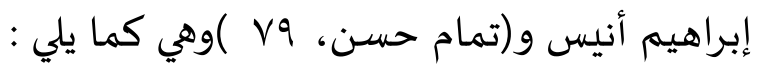



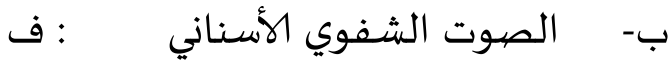

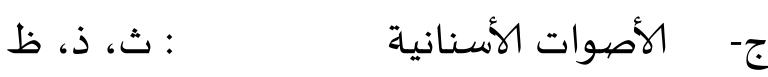

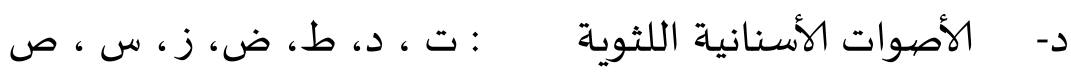

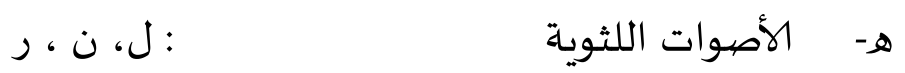

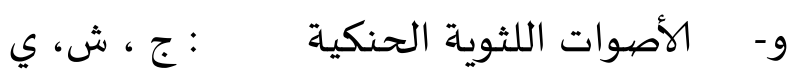

$$
\begin{aligned}
& \text { ز- زأصوات الطبقية }
\end{aligned}
$$

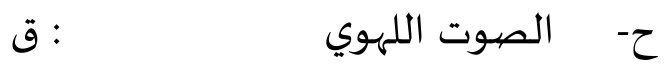

$$
\begin{aligned}
& \text { ط- الأصوات الحلقية }
\end{aligned}
$$

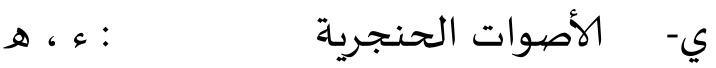

r.) وصف الأصهوات من حيث خروج الهواء أثناء النطق

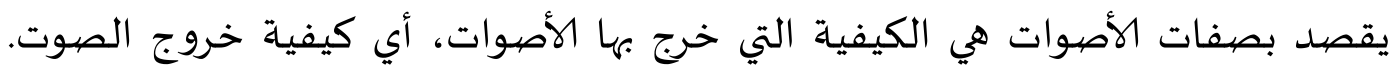

وقال أبو بكر حسيني أنها كل ما من شأنه ان يكسب الصوت اللغوي خاصاميزة خاصة أو جرسا

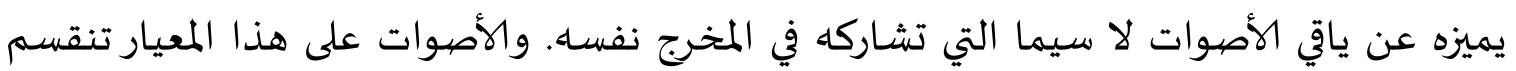

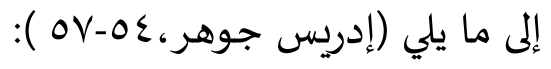

أ- الأصوات الانفجارية، وتسهي أيضا بأصوات شديدة أو أصوات وقفية وهي ثمانية أصوات : ب ،

$$
\text { ت، د، ض، ط، ك، ق، }
$$

ب- الأصوات الاحتكاكية، وتسهى هذا الأصوات أيضا بالأصوات الرخوة أوالأسوات الاستمرارية و هي







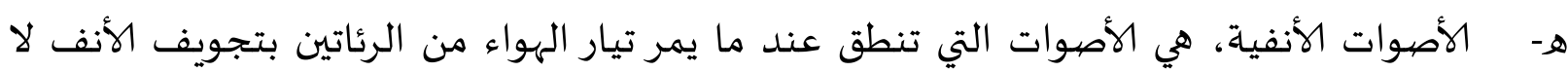

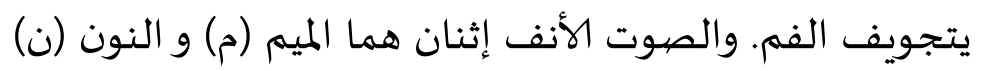

و- الصوت التكرار أو المكرر، هو صوت يحدث عندما يكون التضييق غير ذي استقرارفتكررت

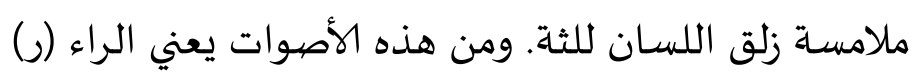

Eva Lathifah Fauzia

اختلافات دراسة علم الأصوات بين العربية واللسانيات الحديثة 
r.) وصف الأصوات العربية من حيث حالة الوترين الصوتيين أثناء النطق(إدريس جوهر،99-ـ .7 ) أ- الأصوات المجهورة، يعني الأصوات التي يهتز الوتران الصوتيان عند نطق بها، مما يعني أن الوترين

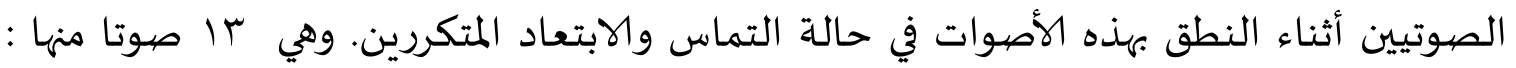

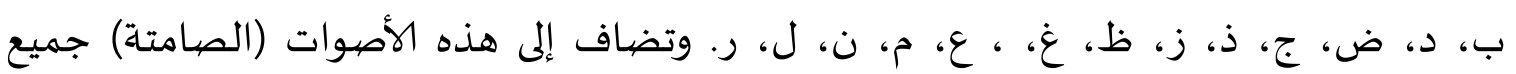

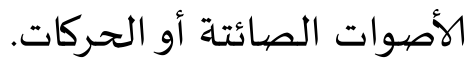

ب- الأصوات المهموسـة، وهي الأصهوات التي لايهتز الوتران الصهوتيان عند النطق بها، مما يعني أن أثناء

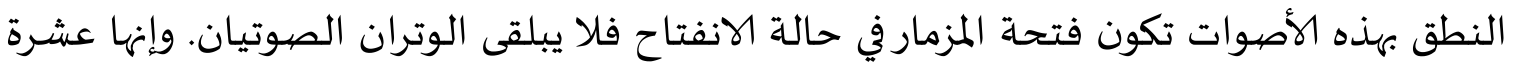

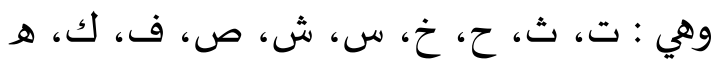

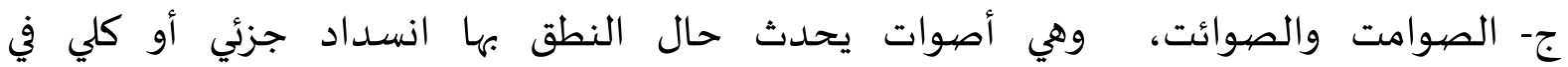

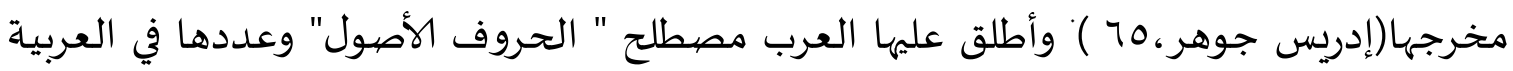

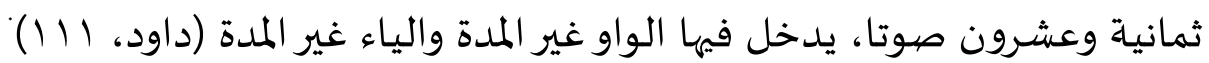

وتعرف الصوائت في العربية بالحركات قصيرة ( الفتحة والكسرة والضمة) بالإضافة إلى

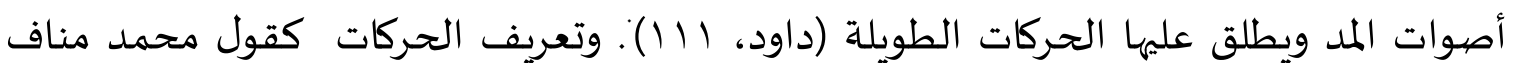
مهدي بإنها أصوات مجهورة يخرج الهواء عند النكق بها على شكل مستمر من البلعوم والفم دون

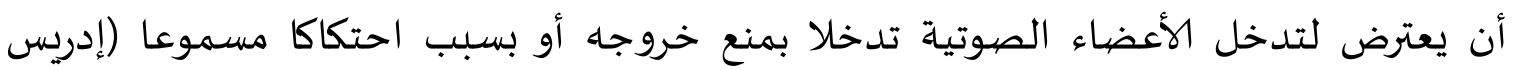
جوهر،70 ). والصوائت أو الحركات في اللغة العربية سته :

i أفتحة القصيرة نحو :لَم

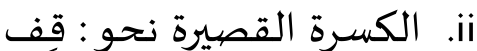

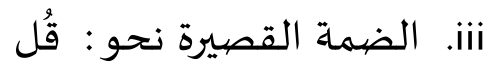

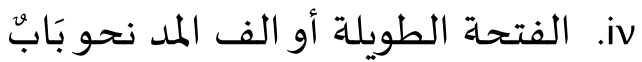

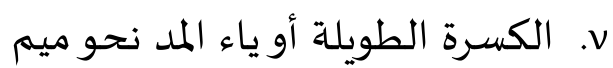

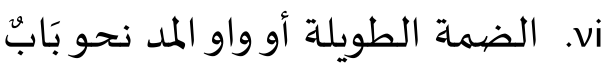

وكل من هذه الحركات الستة لها ثلاث صفات حيث يمكن أن تكون مرققة ومفخمة وبين التفخيم والترقيق.

د- الفونيم أو وحدة صوتية، أن الوحدة الصوتية هي مجموع عناصر نطقية وسمعية لاتقبل التجزئة

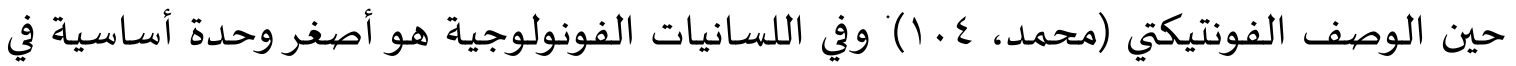

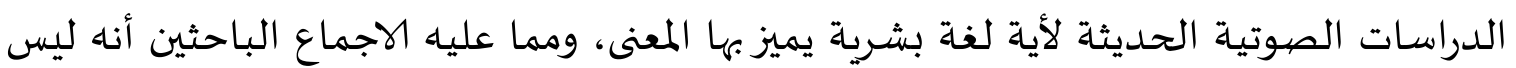

Eva Lathifah Fauzia

اختلافات دراسة علم الأصوات بين العربية واللسانيات الحديثة
ALSUNA Vol. 2 (1), 2019 
للفونيم. وباستثناء الوظيفة أو القيمة الصوتية. أية قيمة أو وظيفة أخرى يؤديها أنيس برا-

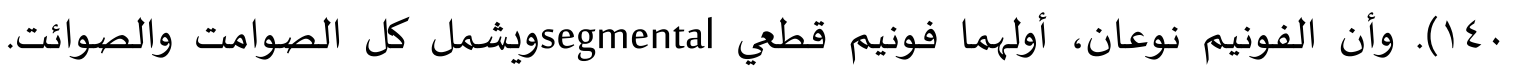
وثانيهما فونيم فوق قطعي وانيتمثل في كل من الفاصل والنغم والنبرة وطول

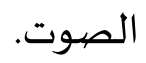

هـ المقطع، ليست الكلمة أصغر وحدة لغوية إذ أنها تتكون من وحدات أخرى أصغر، منها وحدات

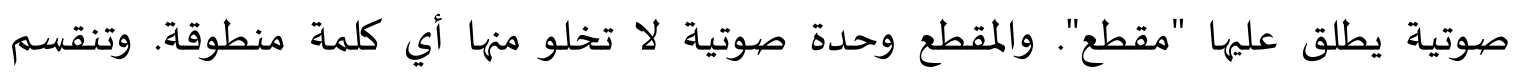

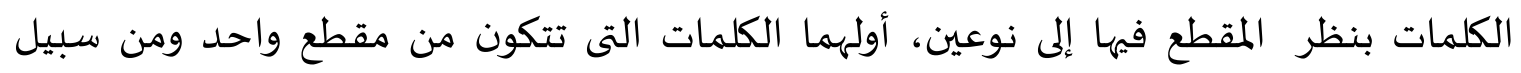

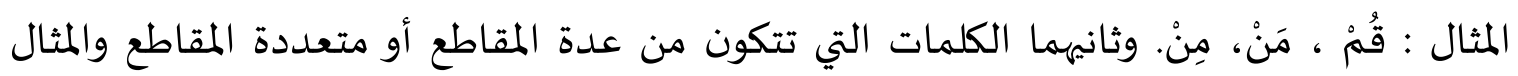

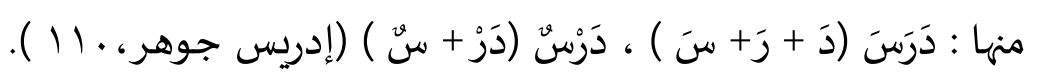

وأثار الباحثون في مجال الأصوات العربية إلى مجموعة من الخواص العامة للمقطع اللغة

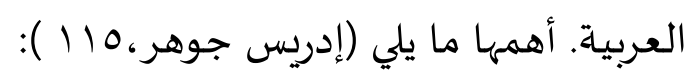

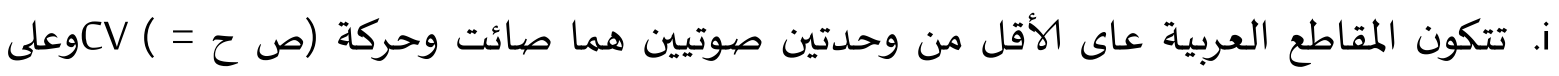

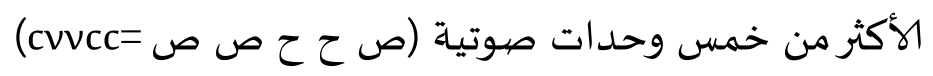

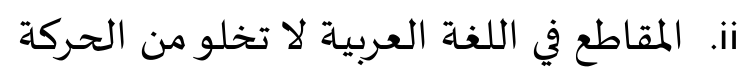

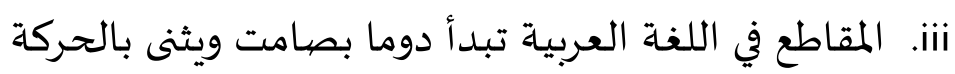

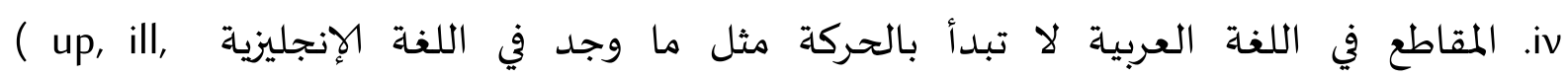

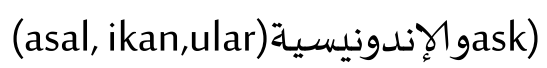

v. المقاطع في اللغة العربية لا تبدأ بصامتين متتاليين مثل ما وجد في الأندونيسية (praktek). و

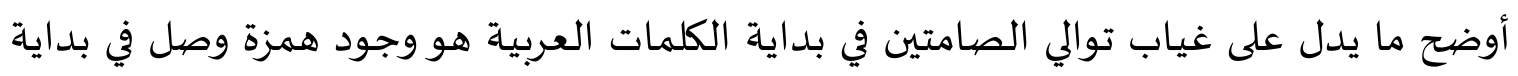

$$
\text { فعل الأمر نخو (اجُلس) بدلا من (جُلس). }
$$

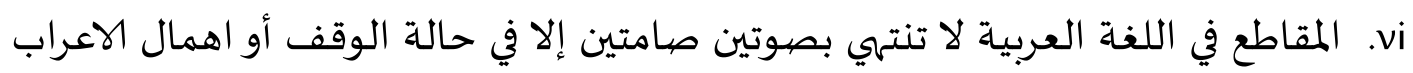

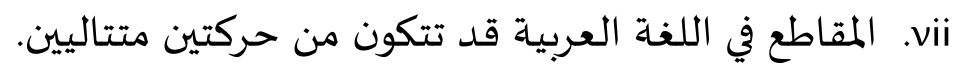

و- النبر، هو نطق مقطع من مقاطع الكلمة بصورة أوضح وأجاى نسبيا من بقية المقاطع التي تجاوره

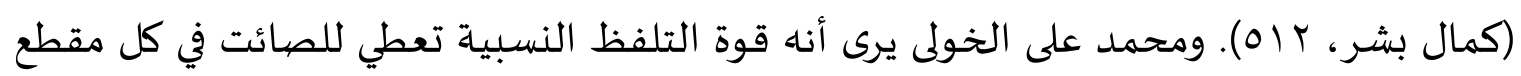

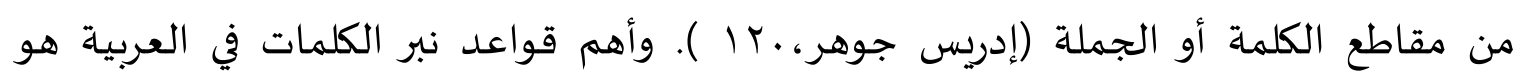

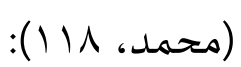

i. يقع النبر في الكلمات الأحادية المقطع على مقطعها الوحيد, نحو : قُمْر ولاً

Eva Lathifah Fauzia

اختلافات دراسة علم الأصوات بين العربية واللسانيات الحديثة 


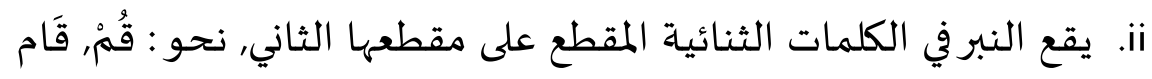

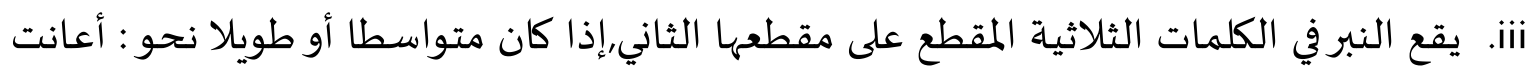

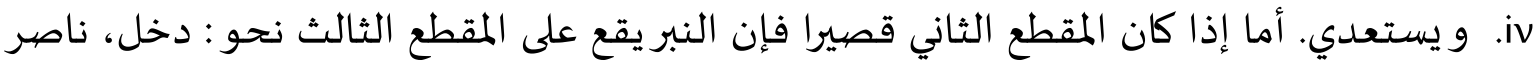
وأما قواعد التبر على مستوى الجملة يقع على القواعد الأتية :

i . يقع النبر على أدةات الاستفهام، والنفي والنهي والشرط. مثال هل قرأت الكتاب ؟

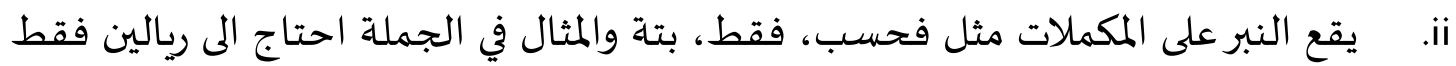

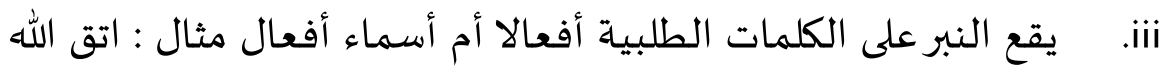

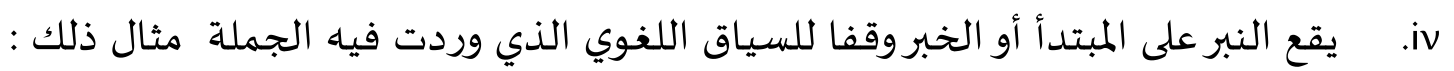

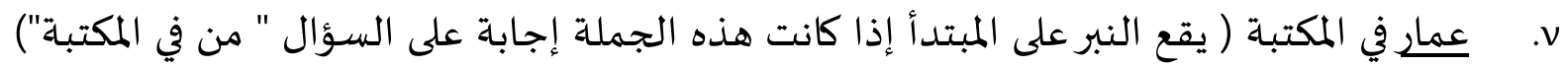

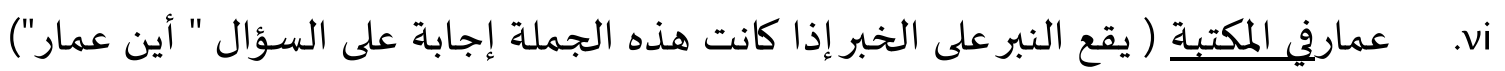
والنبر يقع على أية كلمة من الجملة يراد توكيد معناها.

ز- النغمة والتنغيم، النغمة بأهها درجات الصوت أو طبقاته على مستوى الكلمة ولذلك تسهى تونات الكلمة ـ والنغمة أو تدعى أحيانا "التون" أو درجة الصوت أو طبقة الصهوت هو فونيم فوق قطعي يصاحب نطق فونيمات قطعية ويؤثر في المعنى أو يؤدي إلى تغيير المعنى. وتستعمل النغمة لتمييز

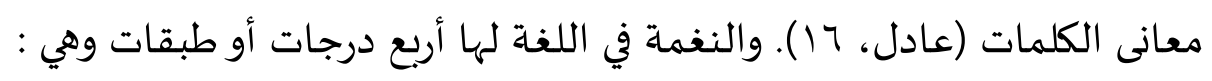

i i. النغمة الهابطة أو المنخفضة وهي النغمة التي تنتهي بها الكلام العادي غير الانفعالي. ومثال ذلك

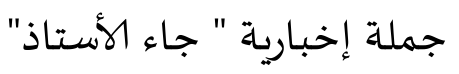

ii

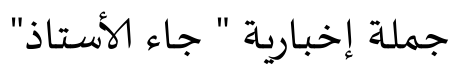

iii النغمة العالية أو المرتفعة وهي النغمة التي تأتي قبل نهاية الكلام وهي متبوعاة بنغمة هابطة.

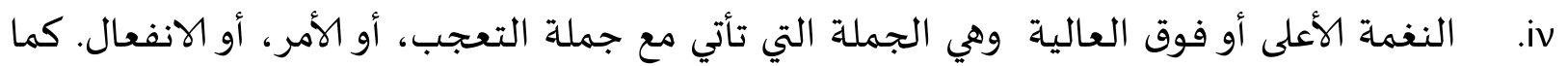

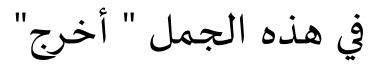

أما التنغيم فهو درجات الصوت على مستوى الجملة أو العبارة أو مجموعة الكلمات (عادل، با ). وقال محمد محمد داود أن التنغيم يطلق على ارتفاع الصيوت وانخفاضها وتلونه بوجوه مختلفة أثناء النطق على المستوى الجملة، وذلك لدلالة على المعان مقصودة، مثل 
الاستفهام، والطلب، والأمر، والغضب، والرضا، والفرح، والدهشة، والتعجب، واللهفة، والشوق

$$
\text { وهلم جر (محمد، سبام (). }
$$

يرى الباحثون في مجال الأصوات أن التنغيم نوع من الفونيمات فوق القطعية (suprasegmental phonems) وقد وضح ذلك كمال بشر لما أشار إلى أن التنغيم يؤدي وظائف في التحليل اللغوي الاتصال

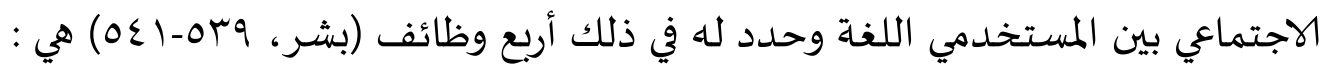
i. الوظيفة النحوية، إن من خلال الأداء الفعلي للتنغيم يمكن تمييز بين الأنماط التركيب والتفريق بين أجناسها النحوياة. الوظيفة الدلالية السياقية، إن الأداء المتباين لأنماط التنغيم في العبارة المعينة يقود إلى التباين

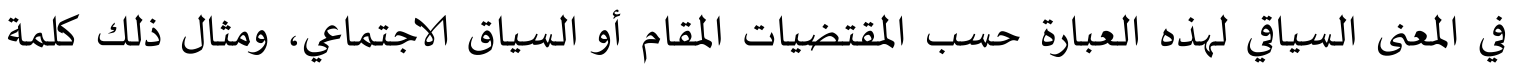
"نعم" قد تحمل معان سياقية مختلفة لذلك تنطق بتنغيمات مختلفة حسب المعنى الذي تنطق لأجله iii. الوظيفة الاجتماعية الثقافية، إن أداء الأنماط المعينة للنتغيم يشير إلى الطبقات الإجتماعية والثقافية المعينة في المجتمع المعين. الوظيفة المعجمية، إن إداء الأنماط المعينة للتنغيم يفرق بين معاني الكلماتعلى مستوى المعجم والنغمة بهذه الوظيفة تدعى نغمة معجمياة.

الخاتمة

علم الأصوات هو علم يبحث عن نظام صوتي من جوانبه مختلفة. ويتفرع علم الأصهوات إلى تفربعات عدبدة. منها علم الأصوات بنظر إلى مادية الأصهوات ووظيفتها ينقسم إلى قسمين هما فونيتيك وفوناميك أو فونولوجيا. ومن حيث منهجه بتفرغ إلى علم الأصهوات الوصفي والمعياري وعلم الأصوات السنكروني والدياكروني وعلم الأصوات المقارن والتقابلى. والتصنيف من حيث ميسرة الأصوات في عملية الكلام ثلاثة هي علم الأصوات النطقي و علم الأصهوات الأكستيكي وعلم الأصوات السمعي. والإطار النظري في علم الأصوات هي : أجهزة النطق، ومخارج الحروف و صفاتها والبحث عن الصوامت والصهوائت، و الفونيم، و المقطع، و النبر، و النغمة والتنغيم ـ وقد يستفيد علم الأصوات في اللغة العربية وتعليمها، أما في اللغة العربية قد ساهم علم الأصوات إلى بياتات للدراسة الصرفية والنحوية والدلالية وكذلك المعجمية. وقد يستفيد علم الأصوات في تعليم العربية بتدريب نطق الأصوات

Eva Lathifah Fauzia

اختلافات دراسة علم الأصوات بين العربية واللسانيات الحديثة 
الصحيح اعتمادا على علم الأصوات النطقي، والتدريب على استعمال الصيوات في التعبير ويكون وظيفة اللغة ، ويعتمد هذا على علم الأصوات التشكيلي او فونيماتيك. ويستفيد من علم الأصوات التقابلي كذلك فى التعليم اللغة العربية حيث تتماثل بين اللغة الأولى والثانية أو بعبارة أخرى لغة الأم و اللغة المدروسة، أو تتشابه بينهما أو تختلف يينهما.

Eva Lathifah Fauzia

اختلافات دراسة علم الأصوات بين العربية واللسانيات الحديثة
ALSUNA Vol. 2 (1), 2019 http://e-journal.ikhac.ac.id/index.php/alsuna https://doi.org/10.31538/alsuna 


\section{Bibliography}

إدريس جوهر، نصر الدين. علم الأصوات لدارسي اللغة العببية من الإندونيسين. لسان عربي, . Y. $1 \varepsilon$

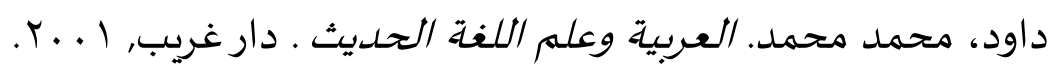

ابن تيمية، أحمد بن عبد الحليم. اقتضاء الصراط المستقيم لمخالفة أصحاب الجحيم.

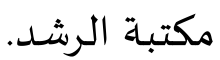

ابن سينا، أبو علي الحسين بن عبد الله. رسالة أسباب حلدوث الحروف، تح: محمد حسان الطيان، يجي مرعلم. مجمع اللغة العربية.

أبو الفتح، عثمان بن جني. الخصيائص ـ دار الكتب المصرية, 1907. الرشيدي، عبد الوهاب. علم الوصوات النطقي. مطبعة جامعة مولانا ملك إبراهيم الإسلامية

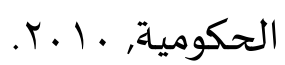

الزمخشري، أبي القاسم محمود بن عمر. المفصل في علم العببية، تح: فخر صالح قدارة. دار

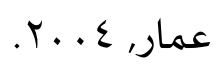

السعران، محمود. علم اللغة، مقلدمة للقارئ العربي. دار الهضية العبية. السكاكي، يوسف بن أبي بكر بن محمد بن علي. مفتاح العلوم. دار الكتب العلمية, 191V. أنيس، إبراهيم. أوصوات اللغوية.نهضة مصر.

أيوب، عبد الرحمن. أصهوات اللغة. مطبعة الكيلاني, 1971. برجشتراسر. التطور النحوي. مكتبة الخانجي, ع 199. حسان, تمام. اللغة العببية معناها ومبناها. دار الثقافة, ع 199. -----. مناهج البحث في اللغة. مكتبة الأنجلو المصرية, . 199. حمود التيهي، صبيح. علم الوصوات عند سيبويه للمستشرق المانمي أرتور شاده. آداب

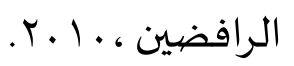

خالف، عادل. أصوات اللغة العبية. المكتبة الأدب, ع199. عبد التواب،رمضان. المدخل إلى علم اللغة ومناهج البحث اللغوي. مكتبة الخانجي, 1999.

Eva Lathifah Fauzia

اختلافات دراسة علم الأصوات بين العربية واللسانيات الحديثة
ALSUNA Vol. 2 (1), 2019 
عثمان بن جني أبو الفتح. سرصناعة الإعراب. تح: حسن هنداوي. دار الكتب العلمياة, . . r. علّوس، جميل. فصول في الثقافة اللنوية. دار الأزمنة, 1999. قدّور، أحمد محمد. مبادئ اللسانيات. دار الفكر, 1997.

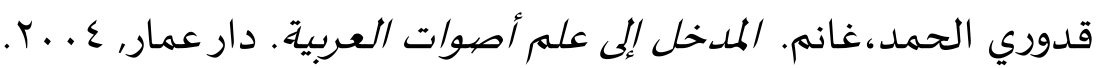
كمال بشر. علم الأصوات. دارغريب, . .. ب.

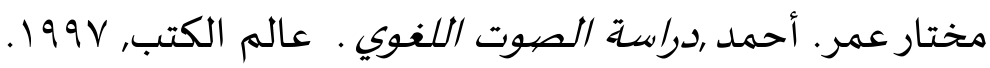
وافي، علي عبد الواحد. علم اللغة. دار نهضاة, .ـ19.

Eva Lathifah Fauzia

اختلافات دراسة علم الأصهوات بين العربية واللسانيات الحديثة
ALSUNA Vol. 2 (1), 2019 http://e-journal.ikhac.ac.id/index.php/alsuna https://doi.org/10.31538/alsuna 